\title{
One step non-contact fabrication of polymer microlens arrays by thermocapillary lithography
}

\author{
Euan McLeod and Sandra M. Troian \\ Laboratory of Interfacial and Small Scale Transport, California Institute of Technology, \\ 1200 E. California Blvd., MC 128-95, Pasadena, CA 91125 \\ stroian@caltech.edu
}

\begin{abstract}
Thermocapillary lithography, a continuous-relief patterning technique, is used to fabricate polymer microlens arrays with variable pitch and focal length. Pattern definition results from sculpting by thermocapillary forces imposed by a cooled mask.

(c) 2009 Optical Society of America

OCIS codes: (220.3740) Lithography; (230.3990) Micro-optical devices
\end{abstract}

\section{Motivation}

Current fabrication methods for microlens arrays and microphotonic structures generally rely on micromolding or hot embossing of polymer films [1]. Direct contact of the mask with the film, however, results in surface roughness and mask degradation from repeated use of the mold. Any further etching introduces additional roughness. Fabrication of the mold itself also requires complex continuous-relief techniques combining photolithographic processing and thermal reflow [1]. Such multistep processes increase manufacturing cost and compromise device quality.

Here we implement a novel thermocapillary lithographic technique to demonstrate microlens array fabrication. This versatile and efficient patterning technique is capable of non-contact, single step, 3D sculpting of molten films by thermocapillary forces. In this work, these forces result from a 3D temperature field imposed by a nearby array of cooled pins, which form the patterned target. Even disparate features can be fabricated side by side in one step. Once the thermal gradients are turned off, the resulting structures solidify in place, revealing ultra smooth interfaces ideally suited for photonic applications. This patterning technique greatly reduces both processing time and cost while extending mask lifetime. It also offers the potential to construct complex 3D shapes not easily attainable by current lithographic methods.

\section{Mechanism for Film Patterning}

The physical mechanism underlying thermocapillary sculpting of nanofilms is based on fluid flow by tangential interfacial stresses generated by variations in interfacial temperature along the air/melt interface. These variations, imposed on the nanofilm by an external cooled target, generate gradients in surface tension which drive flow from warmer to cooler regions. Typically, a cooler patterned target placed in close proximity to a fluid interface will generate very large thermal gradient fields which easily overcome the stabilizing force of surface tension, thereby resulting in guided flow and 3D structure formation. Previously, we have investigated theoretically the characteristics of nanostructure formation using either a flat featureless target [2,3] or a patterned target containing cylindrical pins [4]. The former configuration, identical to the setup in Fig. 1 (a), but without pins, generates an instability leading to spontaneous formation of nanopillar arrays with a characteristic spacing or pitch given by

$$
\lambda_{c}=2 \pi h_{o} \sqrt{\frac{4 \gamma h_{o}}{3 \kappa d_{o} \gamma_{T} \Delta T}}\left(\frac{d_{o}}{h_{o}}+\kappa-1\right),
$$

where $h_{o}$ is the initial film thickness, $\gamma$ is the fluid surface tension, $\kappa$ is the ratio of thermal conductivity of air to fluid, $d_{o}$ is the gap width between the hot and cold substrate, $\gamma_{T}=|d \gamma / d T|$ is the magnitude of the gradient in surface tension with temperature, and $\Delta T$ is the temperature difference within the gap. For polystyrene (PS) films (2.6 kDa) with $80 \leq h_{o} \leq 200 \mathrm{~nm}, 300 \leq d_{o} \leq 2300 \mathrm{~nm}$ and $10 \leq \Delta T \leq 50{ }^{\circ} \mathrm{C}$, spacings $\lambda_{c}$ ranging from $20-70 \mu \mathrm{m}$ have been observed [5]. More importantly, those experiments demonstrate that structure formation is due to thermocapillary forces and not electrostratic attraction by surface charges [6,7] or acoustic phonon radiation pressure [8], as previously believed. Here we demonstrate guided growth and lens formation by exposing a molten PS nanofilm to a prepatterned mask consisting of an array of protruding pins much smaller in diameter than $\lambda_{c}$. Growth triggered in this way is still subject to a lateral deformation scale set by Eq. 1 but with local values of the gap width or temperature difference. 


\section{CML3.pdf}

\section{(a)}

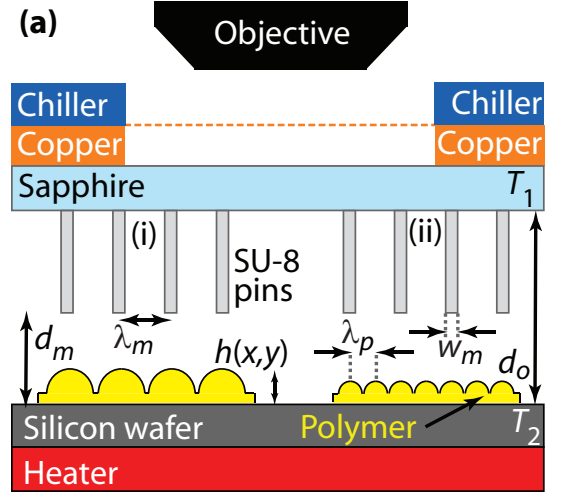

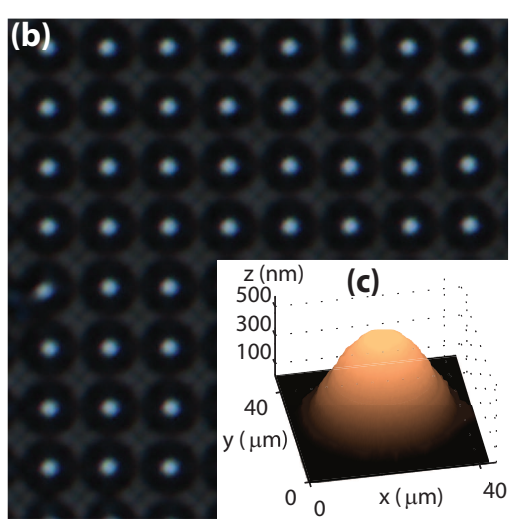

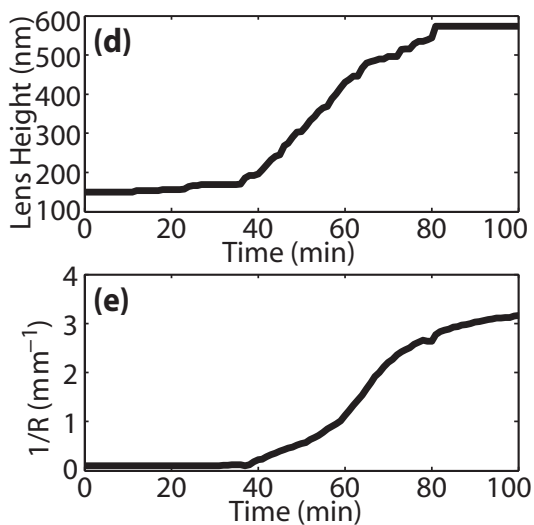

Fig. 1. (a) A patterned cooled mask containing pin-like protrusions triggers formation of microlenses from an initially flat polymer nanofilm. The growth process, terminated before contact with the upper substrate, produces ultra smooth structures with defined pitch and focal length. By varying the experimental parameters, the microarray pitch $\lambda_{p}$ can be made to equal the mask pitch $\lambda_{m}$ or an integral fraction thereof, $\lambda_{p} / n$ (integer n). (b) Defocused microscopy image illustrating microlens focusing. (c) Topography of individual lenslet obtaining by white light interferometry. (d) Peak elevation of lenslet as a function of time. (e) Curvature of a developing lenslet as a function of time.

\section{Tunable Microlens Growth}

Exposing a film to a flat, featureless cooler target as described above generally produces structures with short-range but not long-range order. We find, however, that well-ordered, extended arrays of lenslets can be fabricated easily by pre-patterning a target with protrusions (pins) as shown in Fig. 1(a). These pins project into the air gap and enhance the local thermal gradient imposed on the polymer film, thereby triggering structured formation at specific locations. The resulting structures shown in Fig. 1(b) are able to focus light well. We have also found that pin spacings $\lambda_{m}$ approximately equal to $\lambda_{c}$ result in a microlens array pitch $\lambda_{p}=\lambda_{m}$, as illustrated by Fig. 1(a-i). Values of $\lambda_{m} \gg \lambda_{c}$ generate arrays with pitch $\lambda_{p}=\lambda_{m} / n$ (integer $\mathrm{n}$ ), as illustrated in Fig. 1(a-ii). Pin spacings much smaller than $\lambda_{c}$ tend to form aperiodic arrays with a separation distance of about $\lambda_{c}$. The versatility in setting the array pitch is evident from Eq. 1; critical parameters like $h_{o}$ and $\Delta T$ can be tuned in place without generating a new mask.

In addition to characterizing the optical quality of arrays formed in this way, we also track the in situ growth by optical microscopy and thin-film interferometry. A typical surface topograph is shown in Fig. 1(c) from which the lens curvature and focal length are obtained. Fig. 1(d) shows a typical growth curve for the peak height of a single lens. The growth rate, which scales as $\lambda_{c}^{-4}$, can be adjusted by modifying the controlling parameters in Eq. 1. Fig. 1(e) shows the developing lens curvature as a function of time obtained by fitting the interface to a spherical cap. Lenses with arbitrarily small curvature are easily formed by arresting the growth at a specified time.

Fabrication of polymer microlens arrays by thermcapillary lithography clearly illustrates the simplicity and versatility in forming ultra smooth 3D microstructures by external control of a thermal gradient field. This demonstration paves the way for construction of complex multi-structured features difficult to achieve by conventional photolithography. This work was supported by the CBET division of the National Science Foundation.

\section{References}

[1] H.-T. Hsieh and G.-D. J. Su, "A novel boundary-confined method for high numerical aperture microlens array fabrication,” J. Micromech. Microeng. 20, 035023 (2010).

[2] M. Dietzel and S. M. Troian, "Formation of Nanopillar Arrays in Ultrathin Viscous Films: The Critical Role of Thermocapillary Stresses," Phys. Rev. Lett. 103, 074501 (2009).

[3] M. Dietzel and S. M. Troian, "Mechanism for Spontaneous Growth of Nanopillar Arrays in Ultrathin Films Subject to a Thermal Gradient," J. Appl. Phys. 108, 074308 (2010).

[4] M. Dietzel and S. M. Troian, "Thermocapillary Patterning of Nanoscale Polymer Films," in Materials Systems and Processes for Three Dimensional Micro- and Nanoscale Fabrication and Lithography, S. M. Kuebler and V. T. Milam, eds., 1179E, BB08-02 (2009).

[5] E. McLeod, Y. Liu, and S. M. Troian, "Experimental Determination of Mechanism For Microarray Formations in Nanoscale Molten Films," submitted to Phys. Rev. Lett .

[6] S. Y. Chou, L. Zhuang, and L. Guo, "Lithographically induced self-construction of polymer microstructures for resistless patterning," Appl. Phys. Lett. 75, 1004-1006 (1999).

[7] L. Zhuang, Controlled Self-Assembly in Homopolymer and Diblock Copolymer, Ph.D. thesis, Princeton Univ., Princeton, NJ (2002).

[8] E. Schäffer, S. Harkema, M. Roerdink, R. Blossey, and U. Steiner, "Morphological instability of a confined polymer film in a thermal gradient," Macromol. 36, 1645-1655 (2003). 